\title{
Editorial
}

\section{Juventud y política en sociedades en cambio}

\section{Víctor Muñoz Tamayo ${ }^{1}$, Camila Ponce Lara ${ }^{2}$}

Reflexionar y producir conocimiento sobre el vínculo actual entre juventud y política requiere asumir, de antemano, que se trata de conceptos cuyas dimensiones se encuentran en constante definición, las que no se separan de las propias apuestas de construcción social que establecen los posicionamientos de cada analista.

Por un lado, la política, como ámbito conflictivo de la construcción de sociedad, incluye el debate relativo a entenderla y definirla, con sus límites, vínculos, formas y posibilidades. Como lo sugiere Norbert Lechner (2006), el cientista social puede usar un concepto de política, ligarlo a una determinada concepción de lo social y posicionarse en torno a definiciones, pero ellas no alcanzan la universalidad ni la objetividad, pues se encuentran conectadas con modos y sentidos de acción en tensión y disputa. En resumen, pensar y problematizar la política es parte del hacer

Historiador. Doctor en Estudios Latinoamericanos por la UNAM, México. Investigador del Centro de Investigaciones y Ciencias Sociales y Juventud, Universidad Católica Silva Henríquez, Chile. Contacto: vmunozt@ucsh.cl

2 Socióloga. Doctora en Sociología, École des Hautes Études en Sciences Sociales (EHESS), París, Francia. Investigadora del Centro de Investigaciones y Ciencias Sociales y Juventud, Universidad Católica Silva Henríquez, Chile. Contacto: cponce@ucsh.cl 
político, lo que lo hace necesariamente conflictivo y esquivo al consenso de absolutos.

Por otro lado, la juventud es siempre, como lo planteara Bourdieu (1990), una palabra estructurada socialmente y a la vez estructurante de sociedad, que establece ámbitos, características, distinciones y límites a quienes incluye. Palabra que abarca la pluralidad de modos y condiciones desde las cuales las nuevas generaciones se incorporan a los órdenes e intervienen en su producción y transformación. Y, como agregaran Margulis y Urresti (1996), se trata de un concepto complejo y plural, en el que dimensiones fácticas referidas a lo biológico y lo vital se relacionan en una mutua determinación con lo sociohistórico, lo que hace de las edades construcciones culturales, no naturales ni esenciales.

El siglo XX latinoamericano nos presentó múltiples emergencias de movimientos sociales y militancias ancladas en identidades juveniles que interpretaron la realidad social e intervinieron en ella en términos políticos. Desde el grito antioligárquico de la fundación de la Federación de Estudiantes de Chile FECH (1906), y el reformismo estudiantil de Córdoba (1918), hasta los movimientos estudiantiles de los años sesenta, teñidos de sangre en Tlatelolco, la actoría juvenil fue en gran medida actoría estudiantil. En los años ochenta, las crisis económicas y políticas llevaron las miradas de los analistas a los territorios, donde organizaciones de la juventud pobladora demostraron que la condición estudiantil no era la única realidad a politizar desde identidades que enfatizaban en las vivencias, formas y prácticas juveniles. Hacia los años noventa, los diagnósticos de fragmentación posmoderna y crisis de los grandes relatos; la particular visibilidad que adquirían las culturas y estilos juveniles en el marco de la globalización y las nuevas tecnologías informáticas; la crisis ideológica y política de las izquierdas; el predominio de un estado neoliberal subsidiario respecto al mercado y sus consecuentes procesos de tecnificación administrativa de la política, llevaron a las ciencias sociales a dejar de observar, con la centralidad de antaño, a actores clásicos del siglo XX, como eran 
como los trabajadores y los estudiantes. Esto hasta que, a partir de diversos eventos - desde la huelga larga de la UNAM de México, en 1999, hasta la primavera estudiantil de 2011 en Chile-, los actores juveniles y estudiantiles, con sus particulares condiciones ancladas en el neoliberalismo, remecieron las realidades complejas de nuestros países, produciendo nuevas miradas, prácticas y sentidos relativos a vincular política y juventud.

Aunque en el pasado reciente abundaron los análisis que tendieron a ver en la relación juventud y política una metáfora exagerada de diagnosticadas condiciones de desafección social ante los temas públicos, tales análisis han debido ser revisados ante reiterados ciclos de movimientos sociales juveniles en conflicto con los Estados o los grandes poderes económicos. A ello se suma que ciertas emergencias militantes, con peso significativo de jóvenes generaciones, han alterado los tradicionales esquemas de conformación y confrontación en diversos sistemas de partidos. En definitiva, recientes coyunturas nos dicen que la política, en relación con los jóvenes, desborda las nociones de "apatía", "desafección" o "politicidad" como mera expresividad de descontento por parte de determinadas culturas o estilos juveniles, y que están las condiciones para analizar participación, activismos y militancia juvenil política, lo que supone abordar modos y discursos de politización y de relación entre actores juveniles, Estados y sistemas políticos.

Por lo mismo, la invitación de la presente sección es revisar las formas en que se están produciendo las relaciones entre juventud y política, tarea con la que, de paso, se problematizan tales categorías en relación con lo social. Es también un llamado a unir las perspectivas de los estudios de los movimientos sociales, la protesta y los activismos, con el estudio de las militancias, los partidos y la organización de la política en las sociedades.

Creemos que una distinción útil para entender el carácter de este tipo de problemáticas contemporáneas, es aquella que, en tanto ejercicio analítico, asume una diferenciación entre "la 
política", entendida como campo instituido formalmente, con procedimientos, roles, temas y lógicas específicas (partidos, sistema de partidos, participación electoral), y una dimensión de "lo político" que correspondería al modo cotidiano en que los sujetos asumen la construcción de realidad social y pública. Desde tal mirada, el que "la política" sea continuamente redefinida por temas, conflictividades y dimensiones que emanen de "lo políti$\mathrm{Co}^{\prime \prime}$, sería la condición para que no se separe de lo sociocultural convirtiéndose en un espacio de elite (Garretón 1994, 2004, 2007). Asimismo, el modo en que, desde la sociedad, comienzan a proponerse temas y problemas para ser tratados colectivamente en tanto decisiones públicas, es decir, el modo en que determinados temas pasan al terreno de lo político, constituiría lo que conocemos como procesos de politización de lo social (PNUD, 2015).

La distinción entre lo político y la política resulta útil para el análisis, siempre que entendamos que no hay una separación absoluta, rígida y estática entre las dinámicas asociadas a la acción colectiva de los movimientos sociales, y el ámbito en que las militancias, más o menos instituidas, disputan la conducción del Estado. Entre otras cosas porque las militancias, sean estas más o menos formalizadas, emergen desde dinámicas arraigadas en sujetos sociales insertos en lo político. Basta ver todas las experiencias latinoamericanas en que activismos sectoriales insertos en movimientos sociales se han confundido, imbricado, o han evolucionado hacia militancias políticas con relativa inserción en los sistemas de partidos. Lo anterior ha cambiado el campo de lo político, pero también el de la política, de modo que los cientistas sociales debemos prestar particular atención al terreno de la interrelación de tales dimensiones.

En sintonía con lo anterior, podemos indicar que los trabajos del presente dossier circulan en dos grandes ámbitos conectados. Por una parte, el que se vincula con la acción colectiva contenciosa de actores juveniles en procesos de politización y, por otro, el de actores juveniles politizados que, constituidos en militancias políticas orgánicas, tradicionales o emergentes, actúan desde el 
espectro de las izquierdas o las derechas con más o menos presencia en el sistema político institucionalizado. A continuación, y en consideración a los procesos recientes de politización juvenil en América Latina, daremos una revisión a problemas y temas relevantes presentes en cada una de estas dos dimensiones. Luego de ello, se presentarán los artículos del dossier, relevando el modo en que estos abordan los debates señalados.

\section{Movilizando desde lo político. Protesta, politización y Estado desde la acción colectiva juvenil}

Durante 2011 se genera, a nivel global, una serie de ciclos de protesta que se conocen como "movimientos de plazas" o "novísimos movimientos sociales". Las primeras protestas se originaron en Túnez, contra el gobierno autoritario de Ben Ali, y luego se sumaron países como Egipto, Libia, Siria o Yemen. De manera casi paralela se originaron las movilizaciones en países occidentales contra la crisis económica, política y social. Estos novísimos movimientos sociales (Juris et al., 2012) fueron descritos como diferentes de los "nuevos movimientos sociales" que en su momento trataron sociólogos como Alain Touraine (2006), aquellos que se conformaron en torno a identidades que iban más allá del conflicto capital- trabajo y del clásico movimiento obrero, y que tuvieron causas tan diversas como las de los movimientos ecologistas, feministas y estudiantiles. De acuerdo con algunos analistas, aunque los novísimos movimientos sociales compartían la amplitud cultural de los "nuevos movimientos", tenían como una de sus principales características distintivas un rol preponderante de las nuevas tecnologías y las plataformas online. Por otro lado, se trataba de activismos que también tenían diferencias con las militancias clásicas adscritas o relacionadas con instituciones formales (Pudal, 2011). Para algunos analistas, había en estas experiencias una presencia notable de "otro activismo" (Pleyers, 2010), el llamado "alter-activismo", que se caracteriza 
por compartir valores con eje en el individuo, sus opciones y estilo de vida, las acciones creativas, la utilización de internet y la necesidad de cambiar el mundo a partir de las propias prácticas cotidianas.

Uno de los casos de movilizaciones "novísimas" más significativas en occidente fueron las desarrolladas en España con los denominados "Indignados del 15-M". En este movimiento se realizaron acampadas en la Puerta del Sol, que eran constantemente desalojadas y vueltas a instalar. El adjetivo de los "indignados" en estas movilizaciones españolas nace a partir del libro Indignaos! de Stéphane Hessel (2011), texto que, además, busca reflexionar sobre la necesidad de generar un cambio de mentalidad, contra la indiferencia y a favor de un descontento activo. También emergen movilizaciones de plazas en Nueva York, en la plaza de Occupy Wall Street; en Estambul, en la plaza de Gezi, o en Syntagma en Atenas, con características similares. La particularidad de estas movilizaciones, destacada por autores como Castells (2012), es que todas ellas son horizontales, no hay organizaciones políticas liderando las protestas, ni tampoco se observan líderes permanentes e indiscutidos que las encabecen.

En el caso latinoamericano, podemos observar que hay muchos elementos que se asemejan a aquellas movilizaciones de plazas, pero también encontramos otras particularidades que los distinguen. Por ejemplo, este ciclo latinoamericano tiene la particularidad de ser eminentemente estudiantil, como son aquellas movilizaciones estudiantiles chilenas del 2011, la MANE en Colombia o el \#YoSoy132 en México. En estos casos, vemos que los movimientos se diferencian por contar con líderes carismáticos y estructuras organizativas propias de las universidades, particularmente en Chile y Colombia, pero se asimilan en el tipo de activismo y el rol importante de internet en su organización, que es central por ejemplo en el caso mexicano. De este modo, se realizan investigaciones con el foco puesto en internet y particularmente en las plataformas online como Facebook y Twitter. Por ejemplo, publicaciones que realizan análisis comparados entre 
distintos casos respecto de los usos de internet y la generación de redes (Galindo, 2016), o análisis específicos de Twitter que revelan su institucionalización como medio de comunicación (García et al., 2014), por nombrar algunos.

Más allá del análisis de marcos para la acción colectiva, entre los que destacan en mayor medida los repertorios (Aguilera, 2012; Fernández, 2013), la creatividad de las protestas es una línea importante para dar cuenta de nuevas formas de acción colectiva que se visibilizan con fuerza en el espacio público. Por una parte, las acampadas desarrolladas por una generación juvenil son instancias que permiten crear espacios de experiencia (Pleyers, 2016) y generar vivencias comunes entre los distintos manifestantes; pero también los flash mobs - o multitudes instantáneas-, como los bailes, besatones o corridas por la educación, que conectaban a extraños en pos de un fin común (Ponce y Miranda, 2016), como era la educación gratuita, de calidad y sin lucro, como exigían los estudiante en Chile; o para proteger la educación superior pública y gratuita, en el caso colombiano.

Por último, en 2018 observamos un nuevo momento de movilizaciones juveniles, esta vez con un carácter feminista. Estas movilizaciones se enmarcan principalmente en países iberoamericanos, donde destacan las revueltas de los pañuelazos verdes argentinos, por el aborto libre, seguro y gratuito; el movimiento estudiantil feminista chileno, que se da principalmente en instituciones de educación superior y que exige educación no sexista y el término del acoso; las revueltas contra la condena de "la Manada" en España, y el movimiento \#EleNao contra Bolsonaro en Brasil (Larrondo y Ponce, 2019).

En definitiva, lo social se ha politizado, y ahí han estado identidades constituídas desde la diversidad de mundos juveniles, en la cual la clase, el territorio, la condición estudiantil, las culturas juveniles, el género, la sexualidad, la etnia, intervienen como dimensiones desde las cuales se articula la politicidad juvenil. En ese sentido, vemos que ninguna dimensión de lo juvenil es 
restrictiva en relación a esa diversidad de lugares de identidad, como lo podemos ver en el protagonismo del actor estudiantil, que sigue mostrándose como expresión de agravios y demandas que apelan al conjunto de desigualdades reproducidas al interior de los sistemas educativos, y expresadas en la relación de los y las estudiantes con el Estado, el mercado y la sociedad en su conjunto.

\section{Hacia la política. Generaciones, militancias juveniles y sistema político en la contemporaneidad}

Por mucho tiempo leímos, en los estudios de juventud, el diagnóstico del declive de la participación política militante. La desafección social con la política institucional, expresada en el decaimiento de la participación electoral, así como el declive de los partidos de masas, tuvieron en las nuevas generaciones un ejemplo reiterado, cual metáfora de un devenir en que lo político se distanciaba de la política. En tal contexto, hubo una tendencia en los análisis sociales en destacar lo político y la politicidad de la protesta y los movimientos juveniles, pero no así las orgánicas específicamente político-militantes, más o menos insertas en los sistemas de partidos. Sin embargo, desde hace algunos años, han sido los mismos procesos del continente los que han llevado a los cientistas sociales a observar tránsitos de politización juvenil que han traído consigo ciclos de repartidización, los que, a su vez, han intervenido en los campos políticos nacionales.

Argentina y Chile han sido dos de los países en que determinados contextos han motivado que, desde los estudios de la politización juvenil y los movimientos sociales, se analicen también las militancias emergentes expresadas en tales movimientos (Vommaro, 2015; Vásquez, Vommaro, Núñez y Blanco, 2017; Muñoz y Durán, 2019). Ahí han resultado de particular interés procesos como la emergencia juvenil de izquierdas, derechas y centros, que por un lado recogen tradiciones políticas y las resignifican, 
y, por otro, innovan en los modos, sentidos y lógicas de la acción política. Para América Latina, ha sido particularmente interesante también la observación del proceso español, en el que la crisis de representación de los partidos tradicionales, en el marco del desastre económico, abrió espacios a nuevas militancias que reconocían como anclaje identitario lecturas, indignaciones o desafecciones expresadas por movimientos sociales con presencia juvenil, como es el caso de la relación entre Podemos y el 15-M.

La emergencia de tipos de militancias que aspiran al poder expresado institucionalmente, pero también a ser actor del poder construido desde los movimientos sociales, no son una novedad a la luz del largo siglo XX. Sin embargo, sí lo son en el marco de un neoliberalismo que había conseguido cierto éxito en la tarea de tecnificar la política y despolitizar lo social, en tanto condición básica de los consensos relativos a la aceptación del modelo económico. El 2011 en Chile fue, en ese sentido, la expresión clara de un movimiento juvenil estudiantil que no sólo se asumió político, sino que acusó a la política instituida de no cumplir su tarea de representar políticamente los diversos intereses expresados en la sociedad civil. Denunciando un vacío de conflictividad en el sistema de partidos, el movimiento habló de una democracia deficitaria, atrapada en consensos anquilosados de la elite, que se instalaba como ámbito de administración de lo dado, pero no como canal para las demandas transformadoras emanadas desde la sociedad. En esa línea, no fue extraño que buena parte de las militancias estudiantiles convergieran luego en procesos nacionales de emergencia de nuevos partidos políticos, los que hablaron de cambiar la política desde las propias instituciones, tarea para la cual, según sus propios conceptos, debían ganar representación parlamentaria, apostar por dirigir gobiernos locales, elaborar apuestas en relación a disputar el gobierno nacional, pero sin perder la conexión con el poder de los movimientos sociales.

Al calor de este tipo de transformaciones, los estudios de juventud, política y militancia constituyen ámbitos prioritarios desde los que analizar la conflictiva construcción de lo público 
a partir del foco socioetario y sociogeneracional. Desde ahí se pueden elaborar miradas que parten de la atención en lo juvenil, pero se dirigen a fenómenos que lo trascienden, y que se relacionan con las transformaciones contemporáneas en las conexiones entre Estado, economía y sociedad. La política está cambiando, siempre lo ha hecho, y observar las nuevas generaciones sigue siendo algo fundamental para entender el procesamiento y articulación de esos cambios.

\section{Los artículos de la sección dossier}

Iniciamos la sección dossier con el artículo titulado "Movimientos juveniles en Brasil y México, coordenadas para un análisis de subjetivación y desmovilización social", de Héctor Andrade. Este texto analiza la subjetivación y desmovilización social de los jóvenes activistas de los movimientos \#YoSoy132, Passe Livre, \#TodosSomosPolitécnico y Ayotzinapa, destacando la relación entre la subjetivación, los medios de comunicación y el vínculo con el Estado. A partir de una metodología cualitativa con base en entrevistas, el autor propone una lectura sobre la desmovilización social generada desde el Estado, a partir de distintos dispositivos, como la represión, la vigilancia y la violencia.

Le sigue el texto denominado "Continuidades y rupturas de la protesta universitaria en el Chile de la posdictadura (19902014)", desarrollado por Cristóbal Villalobos y Camila Ortiz. Esta investigación, de carácter descriptivo y cuantitativo, busca estudiar, mediante el análisis de eventos de protesta, los ciclos y tendencias en las movilizaciones estudiantiles del Chile posdictadura entre 1990 y 2014. Los investigadores, a partir de su análisis, persiguen desmontar algunas de las tesis esgrimidas por distintos investigadores que centran su trabajo en el movimiento estudiantil, tales como: la desmovilización durante los noventa y el aumento de conflictividad durante los 2000, el cambio de tácticas y demandas del movimiento estudiantil, y que la intensidad del conflicto ha aumentado durante las últimas décadas. 
Las autoras Mariana Lerchundi y María del Rocío Alonso presentan el artículo "Violencia institucional y participación juvenil: la experiencia de la Marcha de la Gorra (Córdoba)", basado en una investigación sobre la estigmatización y la falta de reconocimiento de jóvenes de las ciudades Río Cuarto y Córdoba en Argentina, en el marco de las movilizaciones de la Marcha de la Gorra. Esta investigación cualitativa, sobre la base de entrevistas en profundidad y etnografías virtuales, busca comprender las significaciones y subjetividades de estos jóvenes fuertemente estimagmatizados.

Por su parte, el investigador Juan Fernández contribuye con "Politización estudiantil y rol de la toma en las movilizaciones de 2011 en Chile". A partir de una metodología de carácter cualitativo, con base en grupos de discusión con activistas de distintas universidades y liceos, el autor busca analizar la relación entre las tomas y la politización de aquellos activistas no dirigentes del movimiento estudiantil de 2011. Uno de los principales hallazgos de este artículo es la apropiación y resignificación de la política por los sujetos jóvenes, además del rol preponderante de la toma como espacio de autonomía y aprendizaje político.

Ya en el tema de las militancias juveniles y su conexión con la política institucional, está el texto de Marion Di Méo titulado: "De la calle al parlamento: trayectorias y repertorios de una generación de estudiantes. Chile, 2006-2017". La autora analiza conexiones entre las movilizaciones de 2006 y 2011, compartiendo la tesis de una continuidad generacional a partir de las vivencias y percepciones de sus actores, primero como estudiantes secundarios y luego como universitarios. Se profundiza en los repertorios de la acción colectiva y su adaptabilidad estratégica, deteniéndose en el tránsito de algunas dirigencias hacia la política institucional.

Una mirada que atiende también al enfoque generacional, pero en un marco histórico de revisión de las trayectorias de militancias juveniles de izquierda durante dictadura, es lo que 
presenta el artículo de Carmen Gemita Oyarzo "Nuestras luchas de ayer: voces militantes y narrativas generacionales sobre la derrota y los desafíos actuales de la izquierda chilena (19902018)". A partir del abordaje del concepto de "generación" en su dimensión de construcción identitaria, es decir, como identidad generacional, Oyarzo hace un riguroso análisis cualitativo de las narrativas de exmilitantes de izquierda, atendiendo al modo en que tales militantes configuran nociones comunes de sentido, en relación a la historia compartida y a las lecturas del presente.

También enfocado en el análisis de la izquierda chilena, el penúltimo texto de la sección dossier lo escribe Aaron Briceño y se titula "El movimiento universitario de Valparaíso y el surgimiento de una nueva izquierda en Chile". Este artículo hace un análisis del movimiento estudiantil universitario de Valparaíso entre 2006 y 2016, centrándose en la izquierda estudiantil y cómo ella se inserta en procesos de articulación de una nueva izquierda en Chile y con presencia en las estructuras formales de representación democrática.

Finaliza esta sección el texto de Rodrigo Torres y Juan Carlos Sánchez titulado "Educación, movilizaciones de estudiantes y conflicto político en Chile y Colombia: algunas reflexiones desde una perspectiva comparada". A partir del análisis de contienda política, con foco en la estructura de oportunidades políticas desarrollada en cada caso estudiado, los autores comparan las movilizaciones estudiantiles recientes de Chile y Colombia, presentando las similitudes en los procesos políticos, así como la evolución de los repertorios de acción de estos jóvenes movilizados.

\section{Referencias}

Aguilera, O. (2012). Repertorios y ciclos de movilización juvenil en Chile (2000-2012). Utopía y praxis latinoamericana: revista internacional de filosofía iberoamericana y teoría social, 101-108.

Bourdieu, P. (1990). La juventud no es más que una palabra. En Sociología y Cultura. México D.F.: Grijalbo/CNCA. 
Castells, M. (2012). Networks of Outrage and Hope. Cambridge: Polity Press.

Fernández, J. (2013). Movimiento estudiantil en Chile (2011): repertorios de acción, marcos de acción colectiva, impactos y desafíos para la política pública. Circunstancia, Revista de Ciencias Sociales del IUIOG, 11(31).

García, C. et al. (2014). What can Twitter tell us about social movements' network topology and centrality? Analysing the case of the 2011-2013 Chilean student movement. International Journal of Organisational Design and Engineering, 4(3-4), 317-337.

Garretón, M. A. (1994). La faz sumergida del iceberg. Estudios sobre la transformación cultural. Santiago de Chile: LOM-CESOC.

Garretón M. A. et al. (2004). América Latina en el siglo XXl. Hacia una nueva matriz sociopolítica. Santiago de Chile: LOM.

Garretón, M. A. (2007). Del postpinochetismo a la sociedad democrática. Santiago de Chile: Debate.

Galindo, L. (2016). La red como cronotopo: Internet y prácticas políticas en el Movimiento Estudiantil Colombiano Mane y Occupy São Paulo. Observatorio (OBS*), 10(Especial), 141-160.

Hessel, S. (2011). jIndignaos! Barcelona: Destino.

Juris, J., Pereira, I. y Feixa, C. (2012). La globalización alternativa y los novísimos movimientos sociales. Revista del Centro de Investigación, Universidad La Salle, 10(37), 23-39.

Larrondo, M. y Ponce, C. (2019). Activismos feministas jóvenes en América Latina. Dimensiones y perspectivas conceptuales. En Activismos feministas jóvenes: Emergencias, actrices y luchas en América Latina. Buenos Aires: CLACSO (En edición).

Lechner, N. (2006). La conflictiva y nunca acabada construcción del orden deseado. En N. Lechner, Obras escogidas, tomo 1. Santiago de Chile: LOM.

Margulis, M. y Urresti, M. (1996). La juventud es más que una palabra. En M. Margulis, La juventud es más que una palabra. Ensayos sobre cultura y juventud (pp. 13-30). Buenos Aires: Editorial Biblos.

Muñoz, V. y Durán, C. (2019). Los jóvenes, la política y los movimientos estudiantiles en el Chile reciente. Ciclos sociopolíticos entre 1967 y 2017. Revista Izquierdas, 45, USACH IDEA. 
Pleyers, G. (2010). Alter-globalization. Becoming actors in the global age. Cambridge: Polity Press.

PNUD. (2015). Desarrollo humano en Chile. Los tiempos de la politización. Santiago de Chile: Programa de Naciones Unidas Para el Desarrollo.

Ponce, C. y Miranda, N. (2016). Redes de confianza online y flash mobs: movilizados por la educación. Observatorio $\left(O B S^{*}\right), 10,161$ 175.

Pudal, B. (2011). Los enfoques teóricos y metodológicos de la militancia. Revista de Sociología, (25), 17-35.

Touraine, A. (2006). Los movimientos sociales. Revista Colombiana de Sociología, 27, 255-278.

Vázquez, M., Vommaro, P., Núñez, P. y Blanco, R. (2017). Militancias juveniles en la Argentina democrática. Trayectorias, espacios y figuras de activismo. Buenos Aires: Imago Mundi.

Vommaro, P. (2015). Juventudes y políticas en la Argentina y en América Latina. Buenos Aires: CLACSO. 